\title{
Electro-Optical Properties of Carbon Nanotubes Obtained by High Density Plasma Chemical Vapor Deposition
}

\author{
Ronaldo D. Mansano, Ana Paula Mousinho \\ Laboratório de Sistemas Integráveis da Escola Politécnica da Universidade de São Paulo, Cidade Universitária, São Paulo, Brasil. \\ Email: mousinho@1si.usp.br
}

Received January $19^{\text {th }}, 2011$; revised March 17 $7^{\text {th }}, 2011$; accepted April $6^{\text {th }}, 2011$.

\begin{abstract}
In this work, we studied the electro-optical properties of high-aligned carbon nanotubes deposited at room temperature. For this, we used the High Density Plasma Chemical Vapor Deposition system. This system uses a new concept of plasma generation: a planar coil is coupled to an RF system for plasma generation. This was used together with an electrostatic shield, for plasma densification, thereby obtaining high-density plasmas. The carbon nanotubes were deposited using pure methane plasmas. Three methods were used for the surface modification of the sample: reference substrate (silicon wafer only submitted to a chemical cleaning), silicon wafer with surface roughness generated by plasma etching, silicon wafer with a thin iron film and silicon wafer with diamond nano powder used as precursor materials. For each kind of silicon wafer surface, the carbon nanotubes were deposited with two different deposition times (two and three hours). The carbon nanotubes structural characteristics were analyzed by Atomic Force Microscope and Scanning Electronic Microscope. The carbon nanotubes electrical characteristics were observed by Raman Spectroscopy and the carbon nanotubes electro-optical properties were analyzed by current vs voltage electrical measurements and photo-luminescence spectroscopy measurements. The photoelectric effect in the carbon nanotubes were determined by photo-induced current measurements. In this work, we obtained carbon nanotubes with semiconductor properties and carbon nanotubes with metallic properties. The electro-optical effects depend strongly on the substrate preparation and the deposition parameters of the carbon nanotubes. The carbon nanotubes are high aligned and show singular properties that can be used for many applications.
\end{abstract}

Keywords: Carbon Nanotubes, CVD Process, Electro-Optical Properties

\section{Introduction}

Carbon nanotubes (CNTs) were discovered in 1991. Remarkable progress has been made in the ensuing 14 years, including the discovery of the two basic types of nanotube (single-wall and multiwall). Great strides have been taken in their synthesis; purification and elucidation of the fundamental physical properties, with important steps are being taken toward realistic practical applications [1]. The CNTs exhibit excellent properties for many applications, including: electrical, electro-optical, optical, optoelectronic, mechanical, electromechanical, magnetic, electromagnetic, chemical, electrochemical, thermal and thermoelectric properties [2].

Carbon nanotubes are a unique and novel material with many interesting properties in the electronic, me- chanical and optical domains. Additionally, their incredible strength (a consequence of the famously strong carbon bond in the tubular structure) poses further advantages in nanomechanic applications. Optically, they exhibit photoluminescence, electroluminescence, photoconductivity, and optical nonlinearity, as well as strong and interesting excitonic effects [3].

The CNTs are optically homogeneous; they possess excellent optical transmittance and good electrical conductivity. The effects of photoluminescence and high absorption of CNTs are attributed to the inter-bands optical transitions $[4,5]$. Moreover, because they are direct band gap materials with very similar effective masses for holes and electrons, they hold great potential for optical detection applications [3].

Carbon nanotubes are generally produced by three 
main techniques, arc discharge, laser ablation and chemical vapor deposition [6]. In this work, the CNTs have been deposited at room temperature by high-density plasma chemical vapor deposition.

The structural characteristics of the CNTs were analyzed by micro-Raman spectroscopy, atomic force microscopy and scanning electronic microscopy $[1,2,7,8]$.

Additionally, the electro-optical properties of the CNTs were analyzed by $\mathrm{I} \times \mathrm{V}$ electrical measurements, photoluminescence spectroscopy measurements and photoinduces current measurements. The carbon nanotubes obtained in this work, are high aligned and can show semiconductor or metallic properties. The surface energy of the sample was changed using two different materials as a precursor (diamond powder and iron film) and samples with a surface roughness obtained by plasma etching. These parameters influence the structural and electro-optical properties of the carbon nanotubes obtained by high-density plasma chemical vapor deposition.

\section{Materials and Methods}

The CNTs were produced in an HDPCVD system (High Density Plasma Chemical Vapor Deposition) that uses a new concept of plasma generation. In this case, a planar coil coupled to an RF system for plasma generation, and an electrostatic shield for plasma densification are used. In this mode, high-density plasmas are obtained. For the ion acceleration another RF system in planar configuration is used as seen in Figure 1. The substrate temperature was not controlled, but measured by a type $\mathrm{K}$ thermocouple, which indicated that the temperature never exceeded $90^{\circ} \mathrm{C}$. For the deposition of the nanotubes, pure methane plasma processes were used.

The HDPCVD system has a main chamber (with a diameter of $35 \mathrm{~cm}$ ) where the electrostatic shield (with a diameter of $30 \mathrm{~cm}$ ) is localized. The electrostatic shield can be heated up to $600^{\circ} \mathrm{C}$ by an infrared heater. The planar coil has been placed in the upper part of the chamber, above a borosilicate window and is covered with an electrode shield. RF power (13.56 MHz) is applied to the planar coil centre and also at the lower electrode (the RF systems are independent).

The vacuum system is formed by a turbo-molecular pump (with nominal outflow of $400 \mathrm{l} / \mathrm{s}$ ) and an auxiliary mechanical pump. The minimum basis pressure that the system can reach is $2 \times 10^{-7}$ Torr. In the HDPCVD system, there are two gas distributors. The main distributor was constructed in the cover of the chamber, where a small homogenization chamber (secondary distributor of gases) exists. The distribution of the gases in the process chamber has been made by a holed ring. The HDPCVD

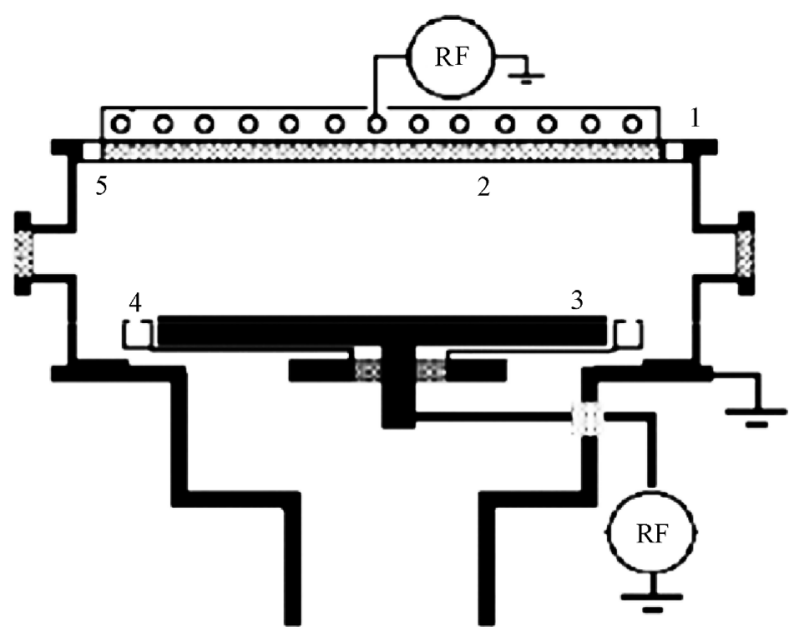

Figure 1. Design of the High Density Plasma Chemical Vapor Deposition system. 1) Planar Coil with an electrode shield, 2) Pyrex window, 3) Electrode, 4) Secondary distributor of gases and 5) Main distributor of gases.

system has been created to work with gases (six different types) and liquids (two different types) at the same time, thus it is possible to obtain DLC films with additives (nitrogen, fluorine, oxygen, etc).

The substrates used to deposit the films were threeinch diameter silicon wafers, $380 \mu \mathrm{m}$ thick and with orientation (100). They were submitted to a Piranha clean, followed by a diluted HF dip, before the modification of the surface. The surface modification is important for the increase of the surface energy of the silicon wafers. Thus, it is possible obtaining CNTs with different structural and electro-optical properties. In this manner, three methods were used for the surface modification of the samples: 1) Diamond powder $(<1 \mu \mathrm{m})$ in alcohol dispersion (deposition by spinner, $1000 \mathrm{rpm}, 30$ section), 2) Iron film deposited by Magnetron Sputtering (5 mTorr, 15 min, $30 \mathrm{sccm}$ of argon) and 3) Roughness generated by plasma etching (RIE system, $\mathrm{SF}_{6}$ plasma, 50 mTorr, 100 $\mathrm{W}, 2$ min.). Besides, the CNTs were deposited in reference substrate (silicon wafers only submitted to a chemical cleaning). For each method, the carbon nanotubes were deposited with two different deposition times: 2 and 4 hours.

The CNTs were deposited after the preparation of the samples, with the parameters: $15 \mathrm{mTorr}, 250 \mathrm{~W}$ (coil power, RF, $13.56 \mathrm{MHz}$, remote plasma), 40-sccm methane. The structural properties of the CNTs were analyzed by Raman spectroscopy. The spectra were collected using a Renishaw micro-Raman 2000 spectrometer on a 40x objective with a photo multiplicator. Unpolarized Raman spectra were acquired at $\lambda=514.5 \mathrm{~nm}$, the spectral resolution was about $4-6 \mathrm{~cm}^{-1}$ and the power on the sample was kept well below $1 \mathrm{~mW}$. The analysis is traditionally car- 
ried out at wavelengths in the blue-green spectral region (about $514.5 \mathrm{~nm}$ ) for analyzing the carbon nanotubes. To calculate the intensities and the areas of the peaks in the Raman spectra, we have made the deconvolution of these spectra (with Gaussian fit), using the Microcal Origin program, with Peak Fitting Mode.

The CNTs were also analyzed by Atomic Force Microscope (AFM). We used an AFM microscope model SPM 9500J3 (Shimadzu). Each sample was analyzed in the AFM in the tapping mode. For each sample, we made ten analyses: five analyses in different areas of $15 \mu \mathrm{m} \times$ $15 \mu \mathrm{m}$ and five analyzes in different areas of $5 \mu \mathrm{m} \times 5$ $\mu \mathrm{m}$. Additionally the CNTs were analyzed by Scanning Electronic Microscope. The Scanning Electronic Microscope used for analyzing the CNTs was a FEI model NOVA nanoSEM 500 microscope.

To analyze the electro-optical properties of CNTs we used a Jobin Yvon-SPEX HR model 460, in the range of 500 to $900 \mathrm{~nm}$, with a laser of $480 \mathrm{~nm}$ and $514 \mathrm{~nm}$; the power used was $0.2 \mathrm{~mW}$ for the photoluminescence measurements. For the $\mathrm{I} \times \mathrm{V}$ measurements, was used a picoamperimeter HP 4140B model, with a microprovator Wentworth labs, extremity of $20 \mu \mathrm{m}$ and halogen lamp of $100 \mathrm{~W}$.

\section{Results and Discussions}

The Raman spectroscopy can explore information of the structure, diameter and electronic properties of the carbon nanotubes [9]. In this work, the Raman spectra obtained to the carbon nanotubes deposited on the different samples topography, showed three frequency ranges: low-frequency range, intermediate frequency range and high-frequency range [2].

The low-frequency range (100 to $350 \mathrm{~cm}^{-1}$ ) is called Radial Breathing Mode (RBM). The RBM is a unique phonon mode, appearing only in carbon nanotubes. Its observation in the Raman spectrum provides direct evidence that a sample contains single-walled carbon nanotubes (SWNTs) [10]. The RBM mode is also related to the diameter distribution of the carbon nanotubes.

Figure 2 shows the Raman spectra for the carbon nanotubes deposited on etched silicon wafers by plasma, silicon wafers with diamond powder, silicon wafers with iron and silicon wafers only submitted to a chemical cleaning (reference), detaching the RBM range of the carbon nanotubes.

Observing Figure 2, it is possible to prove that the final time of the deposition processes and the sample surface preparation before the CNT deposition influence the Raman spectra (peaks position and peaks intensity). The RBM peaks appear in all samples obtained in this work, showing the CNT deposition independent of the final time deposition and the sample surface preparation. How-

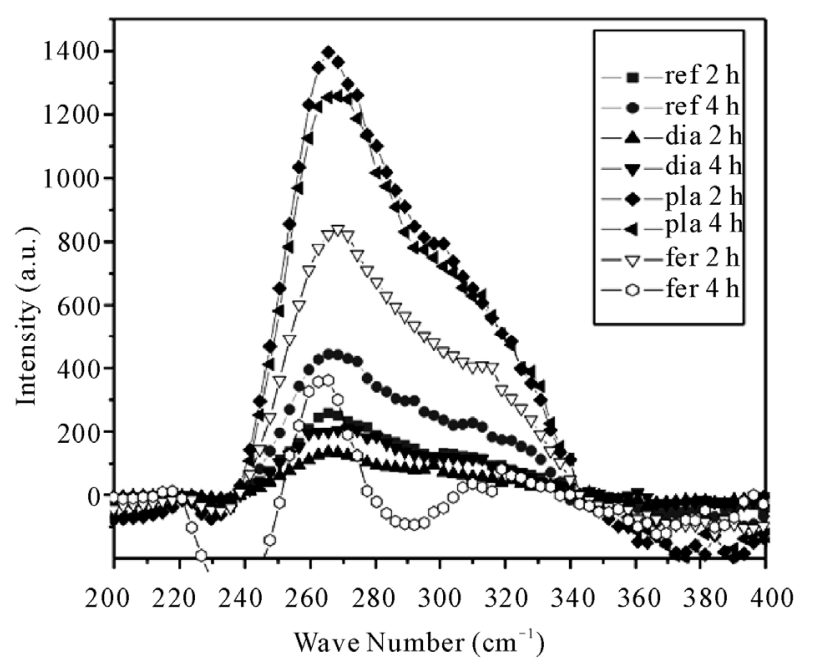

Figure 2. Raman spectra of the CNTs in the RBM region (200 to $400 \mathrm{~cm}^{-1}$ ).

ever, for silicon wafers with roughness generated by plasma etching and under silicon wafer with iron, the peaks related with the RBM appear with high intensity and better definition. For silicon wafers with diamond powder and reference samples, the results were inferior. The strength of the RBM peaks showed less intensity and less definition.

The frequency of the RBM is very sensitive to the nanotube diameter, and the results indicate the influence of the final time deposition and the surface preparation in the structural properties of the CNTs. The G band is an important band in Raman spectrum of the CNTs. Thus, in this work the $\mathrm{G}$ band appeared centered at $\sim 1580 \mathrm{~cm}^{-1}$. The $G$ band is the tangential multi-features at high-frequencies range, as we can see in Figure 3 (typical Raman spectrum of the CNTs obtained by HDPCVD). Observing Figure 3, it is possible to observe others bands in the Raman spectrum. The disorder-induced $\mathrm{D}$ band $\left(\sim 1250 \mathrm{~cm}^{-1}\right)$, the $\mathrm{M}$ band $\left(\sim 1845 \mathrm{~cm}^{-1}\right)$ that is an overtone mode, the iTOLA band $\left(\sim 2230 \mathrm{~cm}^{-1}\right)$ that is a combination of optical and acoustic modes) and the $\mathrm{G}^{\prime}$ band $\left(\sim 2700 \mathrm{~cm}^{-1}\right)$. These bands are related to the singlewalled carbon nanotubes presence, with the intensity of the bands in the Raman spectra related with the final time deposition and the surface silicon wafer before the CNTs deposition.

The M and iTOLA band present in the Raman spectrum indicates that the CNTs show semiconducting features and spectra, whereas without these bands there is indicatation of CNTs with metallic features. In this work, the CNTs deposited on silicon wafers with iron showed metallic features. The CNTs deposited on silicon wafers with diamond powder, on etched silicon wafers by plasma and on silicon wafers only submitted to a chemi- 


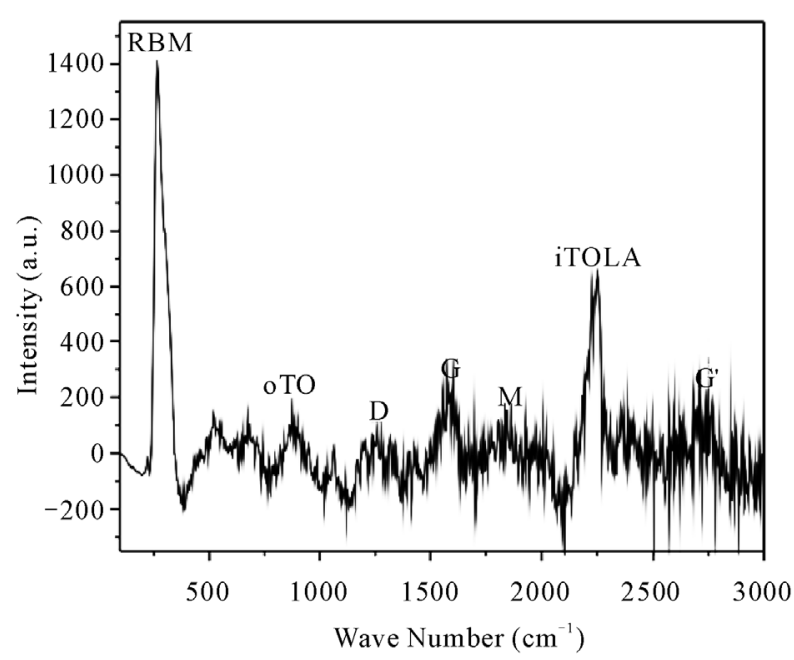

Figure 3. Typical Raman spectrum of the CNTs obtained by HDPCVD (detailing the vibrational modes and bands).

cal cleaning (reference), showed semiconducting features. These results can be confirmed with the electrical measurements of the samples.

Figures 4 and 5, show the AFM images and the SEM micrographs of the CNTs obtained by HDPCVD, respectively. The influence of the surface topography in the structure formation of the CNTs can be observed in these pictures. The CNTs deposited on silicon wafers just submitted a chemical cleaning, are masked due the amorphous matrix presence. This amorphous matrix, formed by special diamond-like carbon (DLC), grows together with the CNTs. In this situation, the growing CNTs occur forming conical islands (as can see in SEM micrograph).

The CNTs growth on silicon wafers etched by plasma and silicon wafers with diamond powder occurs to form sheaves of CNTs. This growth occurs preferentially on the diamond grains and on regions attacked by plasma, respectively. For these samples, the diamond powder presence and the reactive ion etching attack promote surfaces with high roughness. This roughness encourages an increase of the $\mathrm{C}-\mathrm{C}$ bonds, promoting the nanometric structure formation and CNTs growth.

For silicon wafers with iron, the CNTs growth is continuous, forming a film of CNTs. The CNTs deposited on iron are highly aligned and this effect is related to the surface energy of the silicon wafers due the iron presence. In this situation, a preferential region for CNTs growth (as for the other samples used in this work) does not exist. The results can be confirmed analyzing Figure 5.

Figure 6 shows the photoluminescence spectra for CNTs obtained in this work (final time deposition of four hours), for different kinds of silicon surface topographies. The reference sample spectrum shows several fluorescence bands. This effect is due to the presence of other structures. The presence of the amorphous matrix combined with CNTs of different diameters promotes this effect in the photoluminescence spectrum for CNTs deposited on silicon wafers just submitted to a chemical cleaning. A similar effect occurs with CNTs grown on iron. However, in this situation the several bands present are related to CNTs of different diameters. Observing the SEM micrograph of CNTs deposited on silicon wafers with iron, we observed the presence of high-aligned CNTs without carbon amorphous material (as in reference sample). Thus, in this situation the presence of some bands in the photoluminescence spectrum are due to CNTs diameter variation.

The photoluminescence spectra obtained for CNTs grown on silicon wafers with diamond powder and silicon wafers etched by plasma, show high intensity of fluorescence. The increase of surface energy encouraged by the roughness generated by the plasma etching and the diamond powder, generate organized carbon amorphous structures. This effect promotes an emission of a unique band in the photoluminescence spectra and the widening of the fluorescence band due to the CNTs growth in different directions (see Figure 5).

Figure 7 shows the optical absorption spectra for CNTs obtained in this work (final time deposition of four hours), for different kinds of silicon surface topographies. The optical absorption spectroscopy is an excellent tool to study photonic materials because it shows the emission mechanisms in materials, especially CNTs. All samples showed an absorption peak at $1050 \mathrm{~nm}$, characteristic of carbon material films. The most important region in an optical absorption spectrum is the range between $450 \mathrm{~nm}$ and $1000 \mathrm{~nm}$, because this region is related to the CNTs presence. The position of the peaks within this region depends on the chirality and diameter of the CNTs.

Observing the spectra in Figure 7 it is possible to notice the presence of peaks in the range $450 \mathrm{~nm}$ to 1000 $\mathrm{nm}$, and that these peaks are more pronounced with samples deposited on silicon wafers with iron. In this case, the peaks are related to the different diameters of the CNTs present in the samples. The results obtained by Raman spectroscopy and by SEM show the CNTs obtained on iron are highly aligned and well defined. Thus the results obtained by optical absorption spectroscopy confirm the results obtained previously. Within the spectrum acquired for CNTs deposited on samples with iron, there are peaks in the range above of $1050 \mathrm{~nm}$. These peaks are related to the CNT interfaces, promoting emission in different wavelengths.

For the other samples, the presence of the peaks in this region $(450-1000 \mathrm{~nm})$ is tenuous. The results obtained for CNTs deposited on silicon wafers just submitted to a 


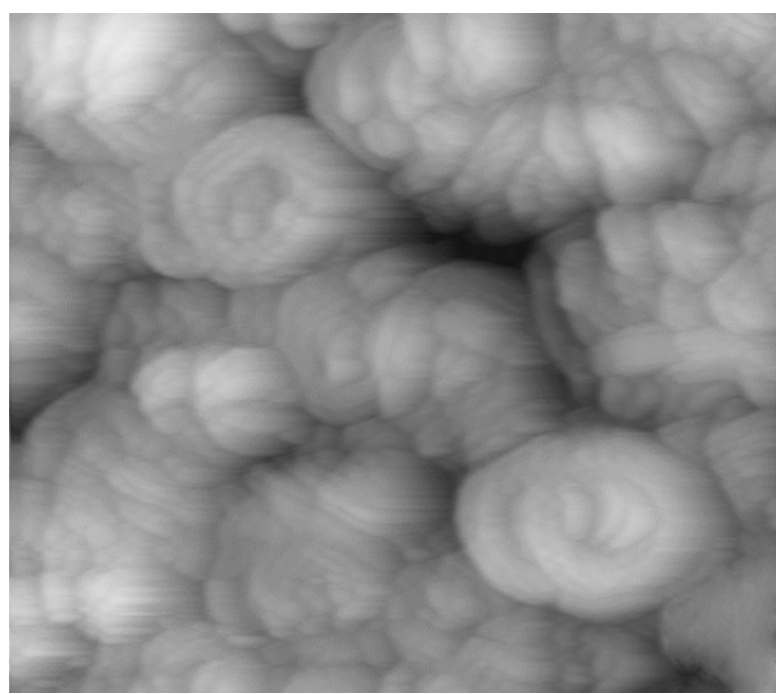

(a)

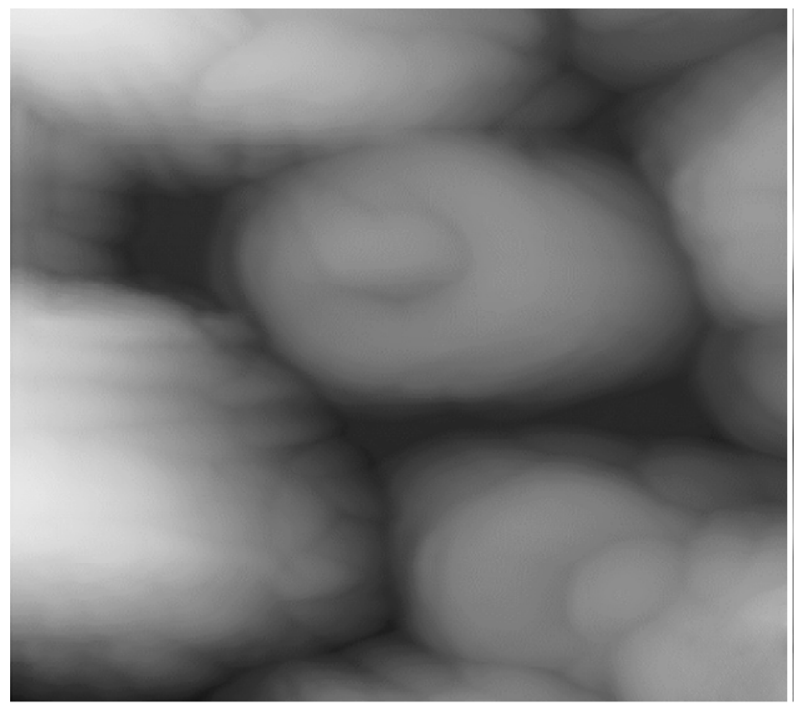

(c)

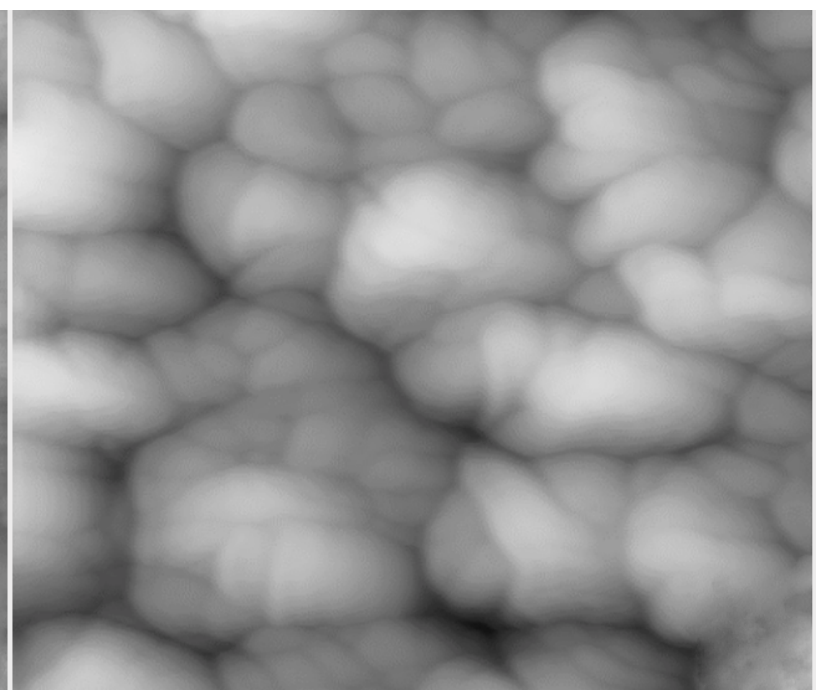

(b)

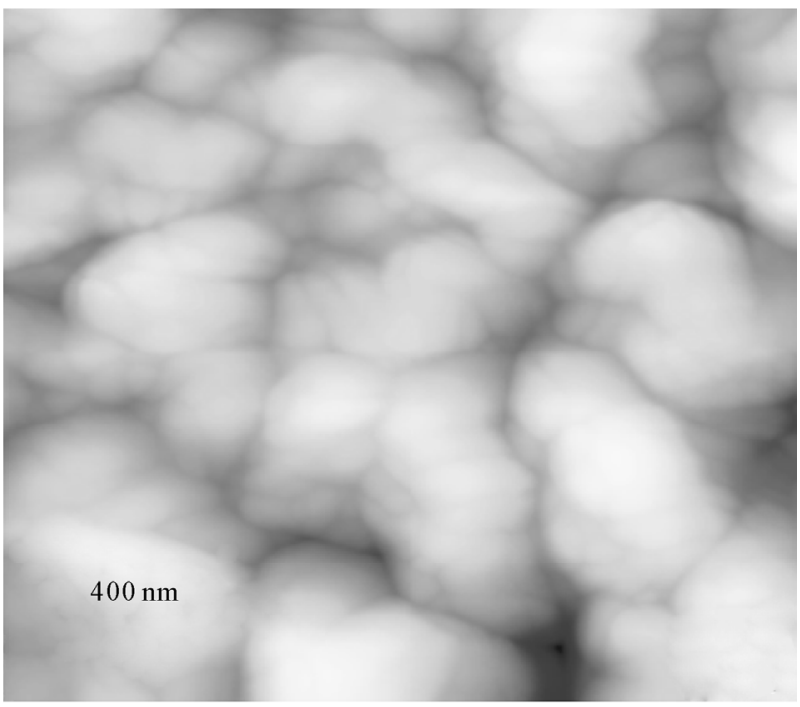

(d)

Figure 4. AFM images of CNTs, (a) silicon wafer just submitted to a chemical cleaning, (b) silicon wafer etched by plasma, (c) silicon wafer with diamond powder and (d) silicon wafer with iron.

chemical cleaning, and on diamond powder and silicon wafers etched by plasma, indicate the CNTs deposition with different diameters, but also indicate the deposition of the other kinds of carbon structures as amorphous carbon, fullerenes, etc. (as can see in Figure 5).

Figures 8 and $\mathbf{9}$, show the results obtained by $\mathrm{I} \times \mathrm{V}$ and photoinduced current measurements for CNTs obtained in this work (final time deposition of four hours); with different kinds of silicon surface topographies respectively. The electrical measurements are important to define the carbon materials that can be used to fabricate phototransistors, photodiodes, etc.

In this work, it was verified that the photoactivity of
CNTs deposited by HDPCVD, when they are exposed to a light source with continuous spectrum. For the electrical measurements of CNTs, we applied an electric potential between $-10 \mathrm{~V}$ and $+10 \mathrm{~V}$, for all samples. In general, as could be seen previously, the CNTs deposited on silicon wafers with iron show metallic properties. Within the results of the $\mathrm{I} \times \mathrm{V}$ measurements it is possible to notice that the proximity between the positive current and the negative current prove the metallic properties of these CNTs. The photoinduced current measurement results prove the metallic character of CNTs deposited on silicon wafers with iron, because there is no evidence of charge injection due to the light effect, as is observed in 


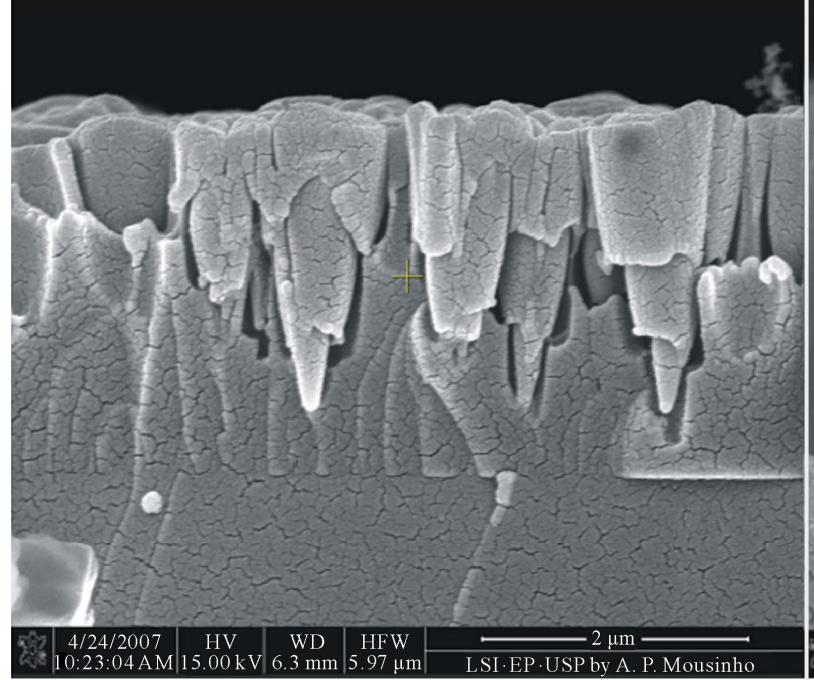

(a)

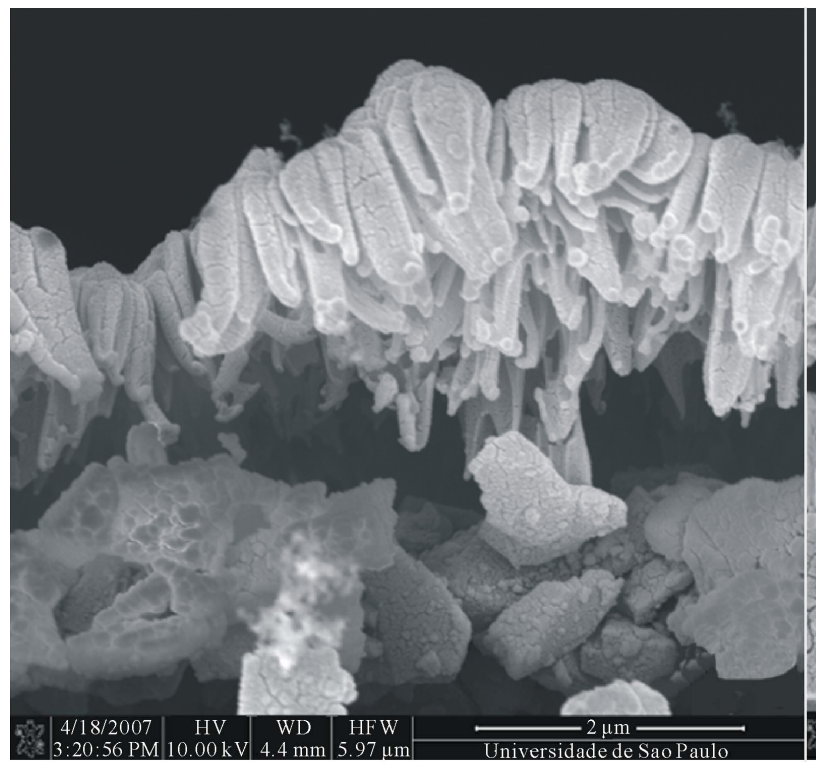

(c)

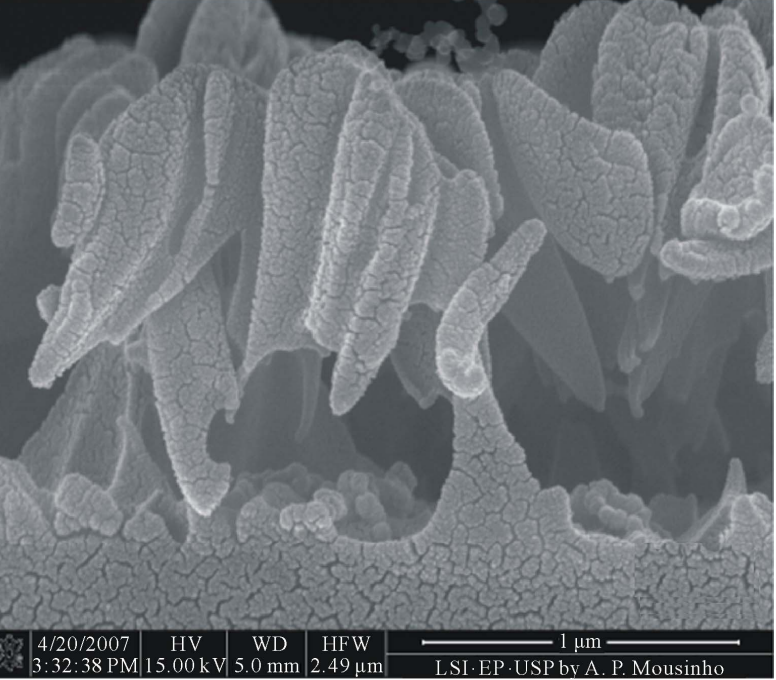

(b)

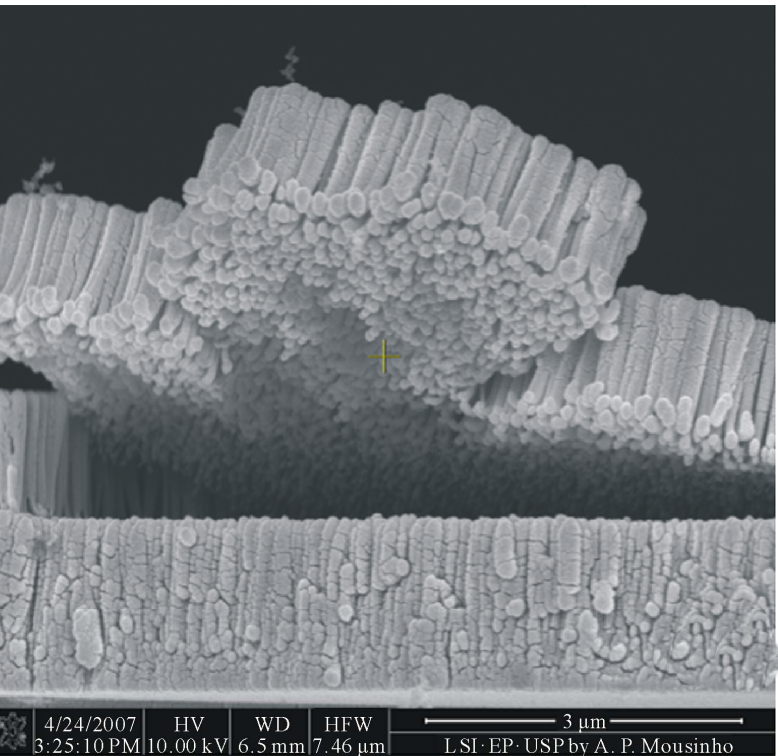

(d)

Figure 5. SEM micrographies of CNTs, (a) Silicon wafer just submitted to a chemical cleaning, (b) Silicon wafer etched by plasma, (c) Silicon wafer with diamond powder and (d) Silicon wafer with iron.

results obtained for the others samples that have semiconducting characteristics.

For highly aligned CNTs (deposited on silicon wafers with iron), the photoinduced current decreases when we used a light source of continuous spectrum and an increase of absorption in the infrared region. These effects occur mainly due to the conduction mechanisms of electrons in CNTs. These effects can be divided into: 1) The jumping between the gaps of the CNTs diameter, especially by the electrons present in the last structures, 2) The tunneling effect between neighboring carbon nanotubes, and 3) The conduction effect along graphene structures. The first two effects are responsible for the emission in the visible spectrum. The first mechanism is responsible for the emission in smaller wavelengths (ultra-violet) and the second mechanism is responsible for the emission in bigger wavelengths. The third mechanism is responsible for modulation and electric conduction along the graphene structure. The third mechanism defines the characteristics of the electric conduction (if metallic or semiconducting) of the CNTs and is also responsible for the emission in the infrared.

Thus, the electro-optical properties of the CNTs deposited in this work show photoconductive response un- 


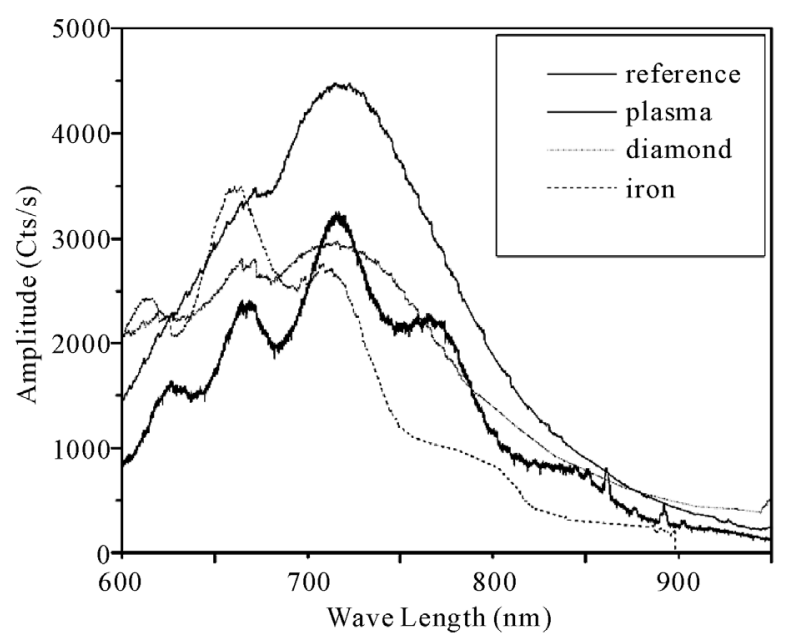

Figure 6. Photoluminescence spectra for CNTs obtained by HDPCVD.
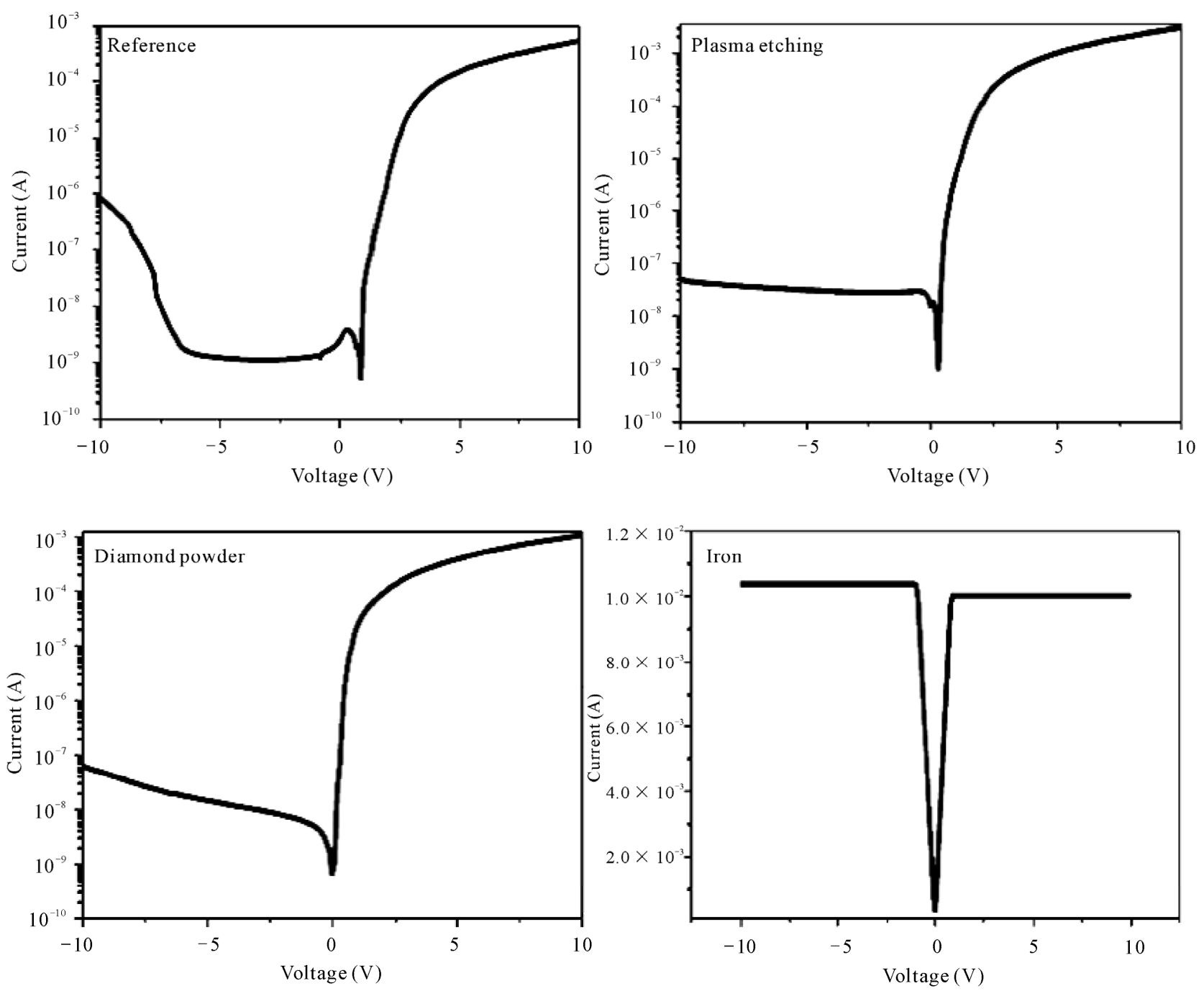

Figure 8. I $\times$ V measurements results for CNTs obtained by HDPCVD. 

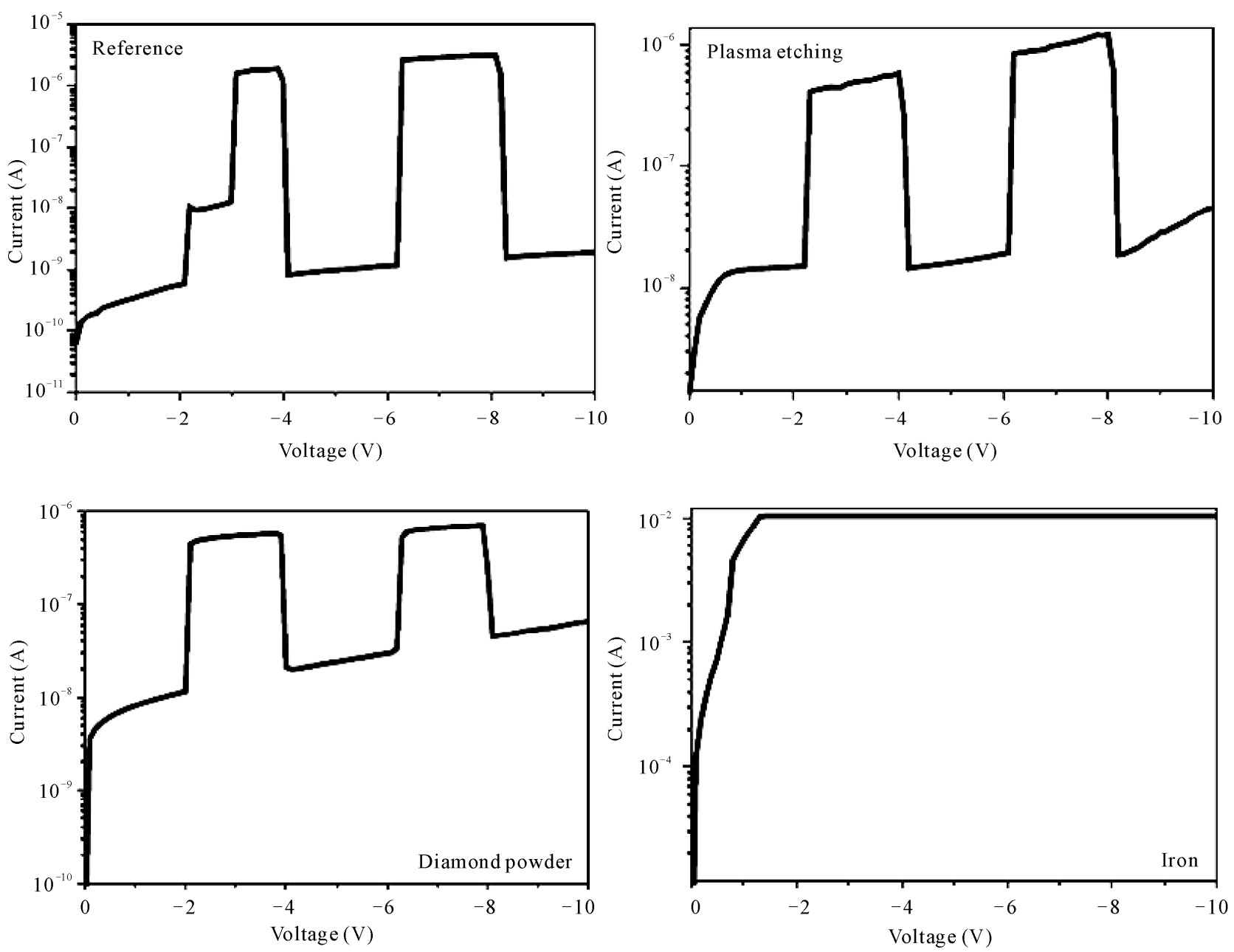

Figure 9. Photo induced current measurement results for CNTs obtained by HDPCVD.

der continuous wave illumination, providing interest not only for a fundamental understanding of the CNT photoinduced process, but also for the development of new photosensitive materials with unique optical and conductive features.

\section{Conclusions}

The main objectives of this work were to: apply CNT deposition by High Density Plasma Chemical Vapor Deposition at room temperature using different surface topographies in silicon wafers, and to the study of the electro-optical properties of CNTs. Using Raman spectroscopy, AFM, SEM, photoluminescence measurements, optical absorption measurements, I vs V measurements, and photoinduced current measurements, it was possible to prove parameters used in the process influence the structural and electro-optical proprerties of the CNTs obtained in this work. The CNTs showed metallic properties when they were deposited on silicon wafers with iron, and showed semiconducting properties when they were deposited on silicon wafers with diamond powder, on silicon wafers only submitted to chemical cleaning, and silicon wafers etched by plasma. With the increase of roughness to the silicon wafers surface by the plasma etching and the diamond powder, the surface energy was changed. This promoted the CNTs growth locally, forming CNT clusters. The CNTs degree of alignment depends on the surface energy of the silicon wafers. The CNTs obtained in this work showed photonic properties, as photoluminescence and optical absorption are influenced by the alignment degree of the CNTs, the diameter of the CNTs, and the quantity of amorphous carbon in the samples. The CNTs deposited on silicon wafers with diamond powder, submitted to a chemical cleaning and etched by plasma, showed semiconducting properties, and the CNTs deposited on silicon wafer with iron showed metallic properties. All CNTs obtained in this work, showed photoactivity with the presence of a photoinduced current with a light source of continuous spectrum. The study of the electro-optical properties permitted the 
proposition of three mechanisms of conduction to CNTs, more distinct with high-alignment degree. These mechanisms are responsible for the electrical and optical properties of the CNTs. This study, demonstrated that the single-walled carbon nanotubes are capable of absorbing infrared light and generating a photocurrent under low applied bias. These results prove the possibility of using the CNTs in photodetectors, photodevices, phototransistors, etc.

\section{Acknowledgements}

The authors would like to thank Dr. Marcos Massi and Mr. Silvio A. de Souza for supporting the AFM analyses, Dr. Patrick B. Verdonck and MSc. Peter L. Polak for supporting this work and Mr. Nelson Ordonez, Mr. Alexandre Camponucci for technical input and support, Mr. Dave Devito for supporting the English corrections, and FAPESP, CAPES and CNPq for financial support.

\section{REFERENCES}

[1] Y. Gogotsi, "Carbon Nanomaterials," CRC Press \& Francis Group, Boca Raton, 2006.

[2] M. Meyyappan, "Carbon Nanotubes, Science and Applications," CRC Press \& Francis Group, Boca Raton, 2004. doi:10.1201/9780203494936

[3] D. A. Straus, M. Tzolov, T. F. Kuo, A. Yin and J. M. Xu, "Quantum Dots and Nanowires-Photocurrent Response of the Carbon Nanotubes-Silicon Heterojunction Array," IET Circuits Devices and Systems, Vol. 1, No. 3, 2007, pp. 200-204. doi:10.1049/iet-cds:20060105
[4] G. L. Zhao, D. Bagayoko and L. Yang, "Optical Properties of Aligned Carbon Nanotubes Mats for Photonic Applications," Journal of Applied Physics, Vol. 99, No. 11, 2006, pp. 114311-114311-5. doi:10.1063/1.2201738

[5] A. Hagen, G. Moss, V. Talalaev and T. Hertel, "Electronic Structure and Dynamics of Optically Excited Single-Wall Carbon Nanotubes," Applied Physics A: Materials Science \& Processing, Vol. 78, No. 8, 2004, pp. 1137-1145.

[6] M. Daenen, R. D. Fouw, B. Hamers, P. G. A. Janssen, K. Schouteden and M. A. J. Veld, "The Wondrous World of Carbon Nanotubes: A Review of Current Carbon Nanotubes Technologies," Multidisciplinary Project Group, Eindhoven University of Technology, Eindhoven, 2003.

[7] J. Robertson, "Diamond-Like Amorphous Carbon," Materials Science and Engineering: R: Reports, Vol. 34, No 4-6, 2002, pp. 129-281. doi:10.1016/S0927-796X(02)00005-0

[8] C. Li and T. W. Chou, "A Structural Mechanics Approach for the Analysis of Carbon Nanotubes," International Journal of Solids and Structures, Vol. 40, No. 10, May 2003, pp. 2487-2499. doi:10.1016/S0020-7683(03)00056-8

[9] L. Alvarez, A. Righi, S. Rols, E. Anglaret and J. L. Sauvajol, "On the Raman Spectrum of Nanobundles of Single Wall Carbon Nanotubes," Groupe de Dynamique des Phases Condenseés, Universite de Montpellier II, Montpellier, 2004.

[10] M. Dresselhaus, G. Dresselhaus, R. Saito and A. Jorio, "Raman Spectroscopy of Carbon Nanotubes," Physics Reports, Vol. 409, No. 2, 2005, pp. 47-99. doi:10.1016/j.physrep.2004.10.006 